\title{
CARACTERIZAÇÃO DA VARIABILIDADE DA FREQUÊNCIA CARDÍACA EM INDIVÍDUOS COM SÍNDROME METABÓLICA
}

\author{
CHARACTERIZATION OF THE HEART RATE VARIABILITY IN INDIVIDUALS WITH METABOLIC SYNDROME \\ CARACTERIZACIÓN DE LA VARIABILIDAD DE LA FRECUENCIA CARDIACO EN PACIENTES \\ CON SIINDROME METABÓLICO
}

Jhennyfer Aline Lima Rodrigues' (Profissional de Educação Física) Gustavo Duarte Ferrari' (Profissional de Educação Física) Igor Alexandre Fernandes ${ }^{1}$ (Enfermeiro)

Letícia Perticarrara Ferezin (Gerontóloga)

Átila Alexandre Trapé (Profissional de Educação Física) Carlos Roberto Bueno Júnior' (Profissional de Educação Física)

1. Universidade de São Paulo, Ribeirão Preto, São Paulo, SP, Brasil.

\section{Correspondência:}

Laboratório de Fisiologia e Metabolismo (LAFEM) - EEFERP, USP. Av. Bandeirantes, 3900, Ribeirão Preto, São Paulo, SP, Brasil. 14040-907.jhenny.jf@gmail.com

\section{RESUMO}

Introdução: A variabilidade da frequência cardíaca (VFC) tem sido considerada um mecanismo de modulação do sistema nervoso autônomo. A diminuição da VFC pode estar associada à síndrome metabólica (SM). Objetivo: Comparar a VFC e variáveis de saúde em indivíduos com e sem SM. Métodos: Cento e dezenove participantes foram divididos em dois grupos: sem SM (SSM, $n=68)$ e com SM (CSM, $n=51)$. Foi avaliada a análise espectral da VFC em repouso, durante teste cardiopulmonar de exercício (TCPE) e na recuperação em bandas de baixa frequência ( $L F=0,04-0,15 \mathrm{~Hz}$ ), alta frequência ( $\mathrm{HF}=0,15-0,4 \mathrm{~Hz}$ ) e razão $L F / H F$. Adicionalmente, a frequência cardíaca $(F C)$ de repouso (FCrep), FC máxima (FCmáx), pressão arterial sistólica (PAS) e diastólica (PAD), glicemia, perfil lipídico, consumo de oxigênio pico $\left(\mathrm{VO}_{2 \text { pico }}\right)$ e composição corporal foram avaliados. Resultados: $\mathrm{A} F C r e p$ e o $\mathrm{VO}_{2 \text { pico }}$ não apresentaram diferenças entre o CSM e o SSM $(73,3 \pm 9,1$ vs. 70,1 $\pm 11,0 \mathrm{bpm})\left(26,8 \pm 4,6 \mathrm{vs} .28,1 \pm 6,6 \mathrm{ml} \cdot \mathrm{kg}^{-1} \cdot \mathrm{min}^{-1}\right)$, respectivamente. AVFC foi similar entre os grupos nos diferentes momentos analisados. A glicemia $(99,8 \pm 22,5$ vs. $87,6 \pm 8,6 \mathrm{mg} / \mathrm{dl})$ foi superior no CSM comparado ao SSM. Os valores de triglicérides $(159,5 \pm 68,8$ vs. 89,2 \pm $34,3 \mathrm{mg} / \mathrm{dl})$ e VLDL-c $(31,9 \pm 13,8$ vs. $17,8 \pm 6,9 \mathrm{mg} / \mathrm{dl})$ foram superiores no CSM comparado ao SSM. O HDL-c ( $40,7 \pm 11,5$ vs. $49,3 \pm 9,8 \mathrm{mg} / \mathrm{dl}$ ) foi menor no CSM comparado ao SSM. O IMC $\left(33,1 \pm 4,7 \mathrm{vs} .30,8 \pm 3,8 \mathrm{~kg} / \mathrm{m}^{2}\right)$ foi superior no CSM comparado ao SSM. A PAS $(128,6 \pm 12,9$ vs. $119,5 \pm 11,3 \mathrm{mmHg})$ e a PAD $(77,2 \pm 10,5 \mathrm{vs} .72,9 \pm 8,1$ $\mathrm{mmHg}$ ) foram superiores no CSM comparado ao SSM, $p<0,05$. Conclusão: Os resultados sugerem que a presença de SM não é suficiente para provocar alterações nos índices de VFC em repouso, durante teste cardiopulmonar de exercício (TCPE) e na recuperação quando os pacientes são comparados a indivíduos sem a doença.

Descritores: sistema nervoso autônomo; obesidade; análise espectral.

\section{ABSTRACT}

Introduction: Heart rate variability (HRV) has been considered a modulation mechanism of the autonomic nervous system. The reduction of HRV may be associated with metabolic syndrome (MS). Objective: To compare the HRV and health variables in individuals with and without MS. Methods: One hundred and nineteen participants were divided into two groups: without MS (WOMS, $n=68)$ and with MS (WMS, $n=51$ ). We evaluated the spectral analysis of HRV at rest, during cardiopulmonary exercise testing (CPET) and recovery in low frequency bands $(L F=0.04-0.15 \mathrm{~Hz}$ ), high frequency $(\mathrm{HF}=0.15-0.4 \mathrm{~Hz})$ and LF/HF ratio. Resting heart rate (HRres), maximum heart rate (HRmax), systolic blood pressure (SBP) and diastolic (DBP), blood glucose, lipid profile, peak oxygen consumption ( $\mathrm{VO}_{\text {2peak }}$ ) and body composition were also evaluated. Results: There were no differences between HRres and $V_{2 \text { peak }}$ between the WMS and WOMS groups (73.3 $\left.\pm 9.1 \mathrm{vs} .70 .1 \pm 11.0 \mathrm{bpm}\right),(26.8 \pm 4.6$ vs. $28.1 \pm 6.6 \mathrm{ml}^{\mathrm{kg}} \mathrm{k}^{-1} . \mathrm{min}^{-1}$ ), respectively. HRV was similar between the groups at the different moments an alyzed. The blood glucose levels $(99.8 \pm 22.5 \mathrm{vs} .87 .6 \pm 8.6 \mathrm{mg} / \mathrm{dl})$ ) were higher in WMS compared to WOMS. Triglyceride values (159.5 $\pm 68.8 \mathrm{vs}$. $89.2 \pm 34.3 \mathrm{mg} / \mathrm{d}$ ) and VLDL-c ( $31.9 \pm 13.8 \mathrm{vs} .17 .8 \pm 6.9 \mathrm{mg} / \mathrm{dl})$ were higher in WMS compared to WOMS. HDL $-\mathrm{c}(40.7 \pm 11.5 \mathrm{vs}$. $49.3 \pm 9.8 \mathrm{mg} / \mathrm{dl})$ was lower in WMS compared to WOMS. BMI $\left(33.1 \pm 4.7 \mathrm{vs} .30 .8 \pm 3.8 \mathrm{~kg} / \mathrm{m}^{2}\right)$ was higher in WMS compared to WOMS. The SBP $(128.6 \pm 12.9 \mathrm{vs} .119 .5 \pm 11.3 \mathrm{mmHg})$ and DBP $(77.2 \pm 10.5 \mathrm{vs} .72 .9 \pm 8.1 \mathrm{mmHg})$ were higher in WMS compared to WOMS, $p<0.05$. Conclusion: The results suggest that the presence of MS is not sufficient to induce changes in HRV at rest, during cardiopulmonary exercise test (CPET), and in recovery when patients are compared to healthy individuals.

Keywords: autonomic nervous system; obesity; spectrum analysis.

\section{RESUMEN}

Introducción: La variabilidad de la frecuencia cardiaca (VFC) ha sido considerada como un mecanismo de modulación del sistema nervioso autónomo. La disminución de VFC puede estar asociada con el síndrome metabólico (SM). Objetivo: Comparar la VFC y variables de salud en individuos con y sin SM. Métodos: Ciento diecinueve sujetos se dividieron en dos grupos: $\sin S M(S S M, n=68)$ y con SM (CSM, $n=51)$. Seevaluó el análisis espectral de la VFC en reposo durante las pruebas de ejercicio cardiopulmonar (PECP) y la recuperación en banda de baja frecuencia ( $(\mathrm{F}=0,04-0,15 \mathrm{~Hz})$, alta frecuencia (HF =0,15-0,4 Hz) y la relación LF/HF. Además, se evaluaron la frecuencia cardiaca en reposo (FCrep), FC máxima (FCmáx), presión arterial sistólica (PAS) y diastólica (PAD), glicemia, perfil lipídico, consumo pico de oxígeno (VO 2pica) y composición corporal. Resultados: $\mathrm{FCrep} \mathrm{y} \mathrm{VO}_{2 \text { pico }}$ no mostraron diferencias entre CSMySSM $(73,3 \pm 9,1$ vs. 70,1 $\pm 11,0$ bpm) $(26,8 \pm 4,6$ vs. $\left.28,1 \pm 6,6 \mathrm{ml}_{\mathrm{kg}} \mathrm{kg}^{-1} \mathrm{~min}^{-1}\right)$, respectivamente. La VFC fue similar entre los grupos en diferentes momentos analizados. La 
glicemia (99,8 22,5 vs. 87,6 $\pm 8,6$ mg/dl) fue mayor en CSM en comparación con SSM. Los valores de triglicéridos (159,5 $\pm 68,8$ vs. 89,2 $\pm 34,3 \mathrm{mg} / \mathrm{dl})$ y VLDL-C (31,9 $\pm 13,8 \mathrm{vs} .17,8 \pm 6,9 \mathrm{mg} / \mathrm{dl})$ fueron más altos en CSM en comparación con SSM. HDL-C (40,7 $\pm 11,5$ vs. 49,3 $\pm 9,8$ mg/dl) fue menor en CSM en comparación con el SSM. El IMC (33,1 $\pm 4,7$ vs. 30,8 \pm $\left.3,8 \mathrm{~kg} / \mathrm{m}^{2}\right)$ fue mayor en CSM en comparación con SSM. LaPAS $(128,6 \pm 12,9 \mathrm{vs} .119,5 \pm 11,3 \mathrm{mmHg})$ y la PAD $(77,2 \pm 10,5$ vs. 72,9 $\pm 8,1 \mathrm{mmHg}$ ) fueron más altas en CSM en comparación con SSM, $p<0,05$. Conclusión: Los resultados sugieren que la presencia de SM no es suficiente para provocar cambios en los índices de VFC en reposo durante las pruebas de ejercicio cardiopulmonar (PECP) y en la recuperación cuando se comparan los pacientes y los individuos saludables.

Descriptores: sistema nervioso autónomo; obesidad; análisis espectral.

\section{INTRODUÇÃO}

Um estilo de vida sedentário associado a uma alimentação inadequada predispõe a um perfil lipídico desfavorável, elevação na pressão arterial (PA), altos níveis de glicose, resistência à insulina e alterações desfavoráveis na composição corporal ${ }^{1}$. Estes fatores de risco ambientais têm papel importante na predisposição à síndrome metabólica (SM), que pode ser definida de acordo com a International Diabetes Federation (IDF) como obesidade central (circunferência cintura $\geq 90 \mathrm{~cm}$ para homens ou $\geq 80 \mathrm{~cm}$ para mulheres) e mais dois dos seguintes fatores: níveis de triglicérides $\geq 150 \mathrm{mg} / \mathrm{dl}$ ou tratamento específico para esta anormalidade lipídica; HDL colesterol < 40 mg/dl em homens, < 50 $\mathrm{mg} / \mathrm{dl}$ em mulheres ou tratamento específico para esta anormalidade lipídica; pressão arterial sistólica (PAS) $\geq 130 \mathrm{mmHg}$, pressão arterial diastólica (PAD) $\geq 85 \mathrm{mmHg}$ ou tratamento para hipertensão arterial; e glicose plasmática de jejum $\geq 100$ mg/dl ou diagnóstico de diabetes mellitus tipo 2.

A SM está associada com morbidade e mortalidade cardiovasculares aumentadas e acomete milhões de pessoas em todo o mundo², sendo a obesidade o componente mais prevalente e que pode alterar o comportamento da função autonômica cardiovascular. Em estudo realizado com 80 indivíduos obesos mórbidos (IMC > 50kg/m²) foi observada uma redução da atividade parassimpática quando comparada a controles não obesos ${ }^{3}$. Esta redução da VFC somada aos demais componentes da SM determinam risco cardiovascular elevado.

Apesar de vários estudos terem investigado a associação dos componentes da SM com a VFC ${ }^{1,4,5}$, comparado os índices de VFC entre indivíduos sedentários e atletas ${ }^{6}$, caracterizado a VFC em coronariopatas e saudáveis ${ }^{7,8}$ e na obesidade ${ }^{9}$, não foi possível encontrar na literatura estudos que compararam a presença de SM definida de acordo com os critérios da IDF com indivíduos aparentemente saudáveis. Diante disso, o principal objetivo do presente trabalho foi comparar índices de VFC em indivíduos classificados com e sem SM de acordo com a IDF, que é o órgão adotado pela Sociedade Brasileira de Cardiologia para caracterização da SM.

\section{MÉTODOS}

Participaram deste estudo homens e mulheres com idade acima de 18 anos, frequentadores de um programa de atividade física de uma universidade pública de Ribeirão Preto (SP), que atenderam aos seguintes critérios de inclusão: IMC acima de $25 \mathrm{~kg} / \mathrm{m}^{2}$ e não apresentar qualquer impedimento à prática de atividade física de ordem cardiológica, muscular ou articular. Foram excluídos indivíduos que apresentaram: angina instável, infarto cardíaco recente (quatro semanas), insuficiência cardíaca descompensada, doença valvular grave, hipertensão arterial descontrolada, falência renal, presença de cirurgias planejadas no período do estudo, abuso de álcool ou droga e que faziam uso de betabloqueadores. Os indivíduos foram classificados com SM adotando-se os critérios preconizados pela IDF, apresentados anteriormente. A amostra final foi composta por 119 indivíduos, alocados em dois grupos: grupo sem SM (SSM, n =68), constituído por indivíduos classificados sem SM e grupo com SM (CSM, n =51), constituído por indivíduos classificados com SM. Todos os participantes desta investigação foram informados textualmente dos possíveis riscos e benefícios quanto aos testes realizados e assinaram o Termo de Consentimento Livre e Esclarecido. A pesquisa foi aprovada pelo Comitê de Ética em Pesquisa da Escola de Educação Física e Esporte de Ribeirão Preto da Universidade de São Paulo em consoante com a Declaração de Helsinki e registrada sob número CAAE 37573114.6.0000.5659.

Os indivíduos foram avaliados com o auxílio de um estadiômetro com precisão de $1 \mathrm{~mm}$ e em seguida a massa corporal (MC) foi obtida por meio de uma balança com precisão de 0,1 kg Omron (Modelo HBF-510 W, Brasil), a partir da qual foi calculado o IMC, definido pela razão entre a $\mathrm{MC}(\mathrm{kg})$ e altura $\left(\mathrm{m}^{2}\right)$, e avaliada a composição corporal (percentual de gordura, gordura visceral e massa muscular) por meio de bioimpedância. A medida de circunferência de cintura (CC) foi avaliada no ponto médio entre o último arco costal e a crista ilíaca, com o indivíduo em pé e no ponto máximo da expiração normal e a circunferência de quadril (CQ) foi avaliada na altura da maior circunferência das nádegas, estando o avaliador ao lado do individuo, utilizando-se fita métrica com precisão de $0,1 \mathrm{~cm}^{10}$, para cálculo da razão cintura/quadril (RCQ).

\section{Aquisição do sinal de variabilidade da frequência cardíaca}

Em uma sala silenciosa mantida a temperatura ambiente entre 21 e $24^{\circ} \mathrm{C}$, a PA foi medida por meio de um aparelho automático de braço Omron (Modelo HEM- 7113, Brasil). Em seguida os indivíduos colocaram uma cinta com transmissor de captação na região do tórax na altura do $5^{\circ}$ espaço intercostal e os sinais foram transmitidos para o monitor cardíaco, onde foram gravados e posteriormente transferidos por meio de uma interface para um computador compatível, para armazenamento e processamento dos sinais (Polar Team² ${ }^{2}$ Finlândia). Após a colocação da fita os indivíduos eram encaminhados para uma maca, onde permaneciam confortavelmente deitados em decúbito dorsal por dez minutos ininterruptos e recebiam orientações sobre os procedimentos de realização do teste, que incluíam: permanecer respirando normalmente, não flexionar e/ou cruzar as pernas e permanecer em silêncio ${ }^{11}$. Após esse momento, os indivíduos foram encaminhados para a esteira. O sinal de VFC foi captado durante todo o teste, com variação entre cinco e 10 minutos entre os participantes e descrito a seguir. Após interrupção do teste cardiopulmonar de exercício (TCPE) os indivíduos permaneciam na esteira em pé durante um minuto para a aquisição dos sinais de recuperação pós teste.

\section{Análise espectral}

A VFC foi analisada utilizando-se o programa CardioSeries (v2.0,http://sites.google.com/site/cardioseries). O programa foi projetado para realizar análise no domínio da frequência, permitindo ajustes na taxa de interpolação $(4 \mathrm{~Hz})$, comprimento do segmento 
(64 a 8192 pontos) e os limites das faixas de frequência (VLF- 0,00 a 0,04/ LF- 0,04 a 0,15/ HF- 0,15 a 0,40) 12. Para análise da VFC, o padrão de seu comportamento foi registrado batimento a batimento, durante todo o protocolo experimental. Para análise dos dados em repouso foram utilizados os intervalos RR consecutivos do $5^{\circ}$ ao $10^{\circ}$ minuto, após ter sido feita filtragem manual, para eliminação de batimentos ectópicos prematuros e artefatos. Para análise dos intervalos RR durante o teste foi utilizado todo o período de teste com variação entre cinco e 10 minutos entre os participantes. Para análise dos dados na recuperação foram utilizados os intervalos RR consecutivos um minuto após a interrupção do TCPE pelos participantes. Na Figura 1 tem-se um exemplo do registro da VFC em um indivíduo do grupo SSM e na Figura 2 um exemplo do registro da VFC em um indivíduo do grupo CSM nos diferentes momentos.

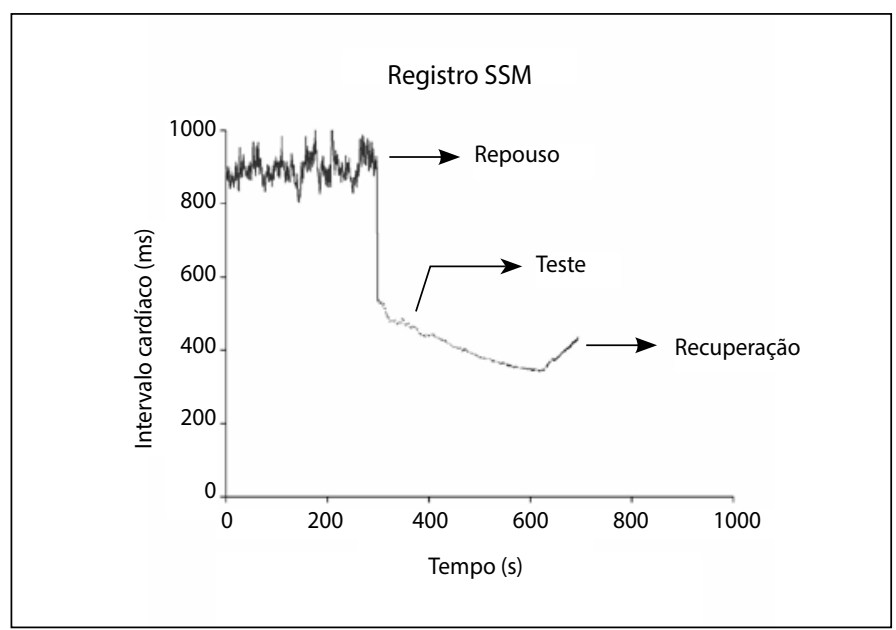

Figura 1. Exemplificação do registro da variabilidade da frequência cardíaca (VFC) em um indivíduo do grupo sem síndrome metabólica (SSM).O eixo X refere-se ao comprimento do segmento analisado (Tempo). $O$ eixo Y refere-se ao intervalo entre cada batimento ( $\mathrm{ms}$ ).

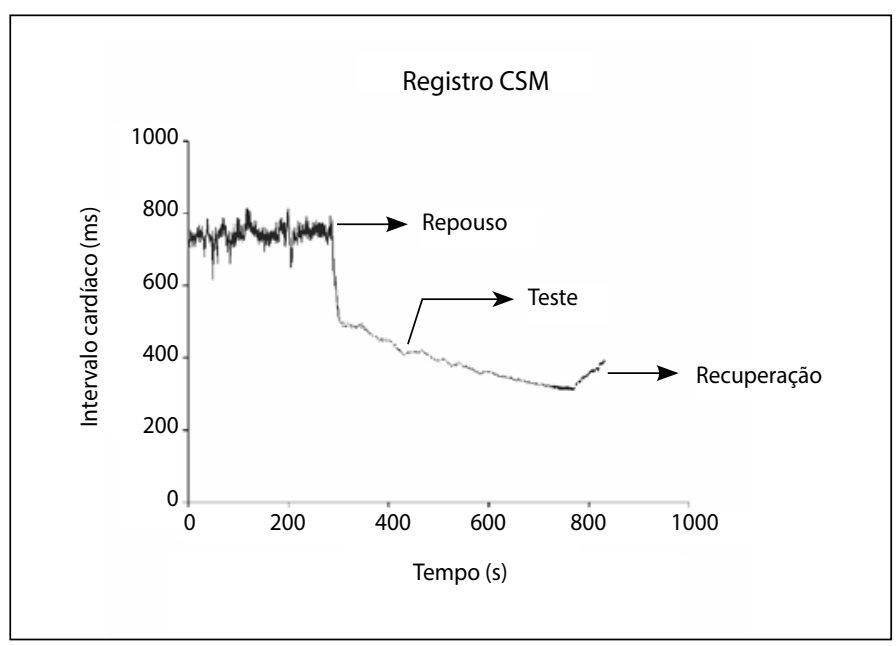

Figura 2. Exemplificação do registro da variabilidade da frequência cardíaca (VFC) em um indivíduo do grupo com síndrome metabólica (CSM).O eixo X refere-se ao comprimento do segmento analisado (Tempo). O eixo Y refere-se ao intervalo entre cada batimento (ms).

\section{Teste cardiopulmonar de exercício}

Imediatamente após o repouso, os indivíduos foram encaminhados para uma esteira rolante motorizada (Inbra Sport Super ATL, Porto Alegre, Brasil) para a realização do teste cardiopulmonar de exercício (TCPE), seguindo protocolo previamente descrito ${ }^{13}$. As variáveis ventilatórias foram mensuradas utilizando o analisador metabólico de gases Quark Ergo PFT (Cosmed, Itália).

\section{Análise sanguínea}

A coleta de sangue foi realizada em jejum de 12 horas por um enfermeiro. Foram coletados cinco mililitros de sangue da fossa antecubital em tubo específico (BD Vacutainer, Estados Unidos). As análises foram realizadas no Laboratório de Análises Clínicas da Faculdade de Ciências Farmacêuticas de Ribeirão Preto por meio de kit de análise enzimática (Wiener Lab, Argentina) em aparelho automático (Konelab, 600i, Argentina).

\section{Análise estatística}

O teste $t$ de Student foi utilizado para comparação das médias entre os grupos. A significância estatística foi aceita quando a probabilidade de erro era menor que o valor alfa fixado em 5\%. A análise dos dados foi realizada com o auxílio do programa Sigma Stat versão 3.5 .

\section{RESULTADOS}

A Tabela 1 apresenta as características dos parâmetros de saúde dos participantes. É possível observar que indivíduos com SM apresentam maior IMC, gordura visceral, CC, RCQ e variáveis sanguíneas alteradas comparadas aos indivíduos sem SM.

A Tabela 2 apresenta os valores médios relativos aos parâmetros cardíacos e ventilatórios, obtidos do registro dos batimentos cardíacos durante a permanência em repouso dos voluntários na posição supina e durante o teste cardiopulmonar de exercício. Não foi possível evidenciar diferenças estatísticas entre os grupos, com exceção para PAS e PAD.

A Tabela 3 apresenta os índices de VFC analisados no domínio da frequência. O componente LF relaciona-se à ativação simpática, o componente HF à ativação parassimpática e a razão LF/HF relaciona-se ao balanço simpato-vagal. Não foi possível evidenciar diferenças entre os grupos nos momentos repouso, no teste cardiopulmonar de exercício e na recuperação.

Na Tabela 4 é possível observar os intervalos cardíacos mínimo, médio e máximo nos três diferentes momentos analisados: repouso, durante o teste cardiopulmonar de exercício e na recuperação. O intervalo cardíaco representa o tempo de duração de um ciclo cardíaco. Não houve diferenças entre os intervalos no repouso, no TCPE e na recuperação entre os grupos. Pode-se observar que com a progressão do TCPE os intervalos RR diminuem em virtude do aumento da demanda cardiovascular para manutenção do esforço.

Tabela 1. Características basais entre indivíduos classificados com e sem síndrome metabólica.

\begin{tabular}{c|c|c|c}
\hline & $\begin{array}{c}\text { SSM } \\
(\mathbf{n}=68)\end{array}$ & $\begin{array}{c}\text { CSM } \\
(\mathbf{n}=51)\end{array}$ & Valor de $\mathbf{p}$ \\
\hline Idade (anos) & $41,2 \pm 10,0$ & $42,8 \pm 10,7$ & 0,466 \\
\hline IMC (Kg/m²) & $30,8 \pm 3,8$ & $33,1 \pm 4,7^{*}$ & 0,003 \\
\hline Gordura (\%) & $42,0 \pm 7,0$ & $41,4 \pm 6,7$ & 0,635 \\
\hline Gordura Visceral (cm²) & $10,3 \pm 3,6$ & $12,8 \pm 5,5^{*}$ & 0,006 \\
\hline Massa Muscular (\%) & $25,6 \pm 4,4$ & $25,9 \pm 4,2$ & 0,678 \\
\hline CC (cm) & $93,1 \pm 11,0$ & $100,3 \pm 12,4^{*}$ & 0,001 \\
\hline RCQ & $0,82 \pm 0,07$ & $0,87 \pm 0,08^{*}$ & 0,001 \\
\hline Glicemia (mg/dl) & $87,6 \pm 8,6$ & $99,8 \pm 22,5^{*}$ & $<0,001$ \\
\hline Triglicérides (mg/dl) & $89,2 \pm 34,3$ & $159,5 \pm 68,8^{*}$ & $<0,001$ \\
\hline Colesterol Total (mg/dl) & $187,9 \pm 32,8$ & $190,2 \pm 33,3$ & 0,711 \\
\hline Colesterol-HDL (mg/dl) & $49,3 \pm 9,8$ & $40,7 \pm 11,5^{*}$ & $<0.001$ \\
\hline Colesterol-LDL (mg/dl) & $116,9 \pm 36,6$ & $117,6 \pm 30,1$ & 0,608 \\
\hline Colesterol-VLDL (mg/dl) & $17,8 \pm 6,9$ & $31,9 \pm 13,8^{*}$ & $<0,001$ \\
\hline $\begin{array}{c}\text { IPAQ - Caminhada } \\
\text { (minuto/semana) }\end{array}$ & $40,8 \pm 72,5$ & $42,9 \pm 80,0$ & 0,973 \\
\hline $\begin{array}{c}\text { IPAQ - AF moderada } \\
\text { (minuto/semana) }\end{array}$ & $30,2 \pm 64,7$ & $33,2 \pm 69,1$ & 0,908 \\
\hline $\begin{array}{c}\text { IPAQ - AF vigorosa } \\
\text { (minuto/semana) }\end{array}$ & $4,7 \pm 21,6$ & $5,3 \pm 19,7$ & 0,298 \\
\hline
\end{tabular}

Valores expressos em média $\pm D P$. * ${ }^{p}<0,05$ vs SSM. SSM - grupo sem síndrome metabólica; CSM - grupo com síndrome metabólica; IMC -índice de massa corporal; CC -circunferência de cintura; RCQ - razão cintura/quadril; $\mathrm{HDL}$ - high densitylipoprotein; LDL - lowdensitylipoprotein; VLDL -verylowdensitylipoprotein; IPAQ - Questionário Internacional de Atividade Física; AF - atividade física. 
Tabela 2. Parâmetros cardíacos e ventilatórios basais e de esforço entre indivíduos classificados com e sem síndrome metabólica.

\begin{tabular}{c|c|c|c}
\hline & $\begin{array}{c}\text { SSM } \\
(\mathbf{n = 6 8 )}\end{array}$ & $\begin{array}{c}\text { CSM } \\
(\mathbf{n = 5 1 )}\end{array}$ & Valor de $\mathbf{p}$ \\
\hline FCrep (bpm) & $70,1 \pm 11,0$ & $73,3 \pm 9,1$ & 0,194 \\
\hline FCmáx (bpm) & $175,4 \pm 13,7$ & $173,0 \pm 17,3$ & 0,423 \\
\hline FCrec (bpm) & $135,4 \pm 14,1$ & $136,4 \pm 18,3$ & 0,748 \\
\hline PASrep (mmHg) & $119,5 \pm 11,3$ & $128,6 \pm 12,9^{*}$ & 0,001 \\
\hline PADrep (mmHg) & $72,9 \pm 8,1$ & $77,2 \pm 10,5^{*}$ & 0,028 \\
\hline $\mathrm{VO}_{\text {2pico }}\left(\mathrm{ml} \cdot \mathrm{kg}^{-1} \cdot \mathrm{min}^{-1}\right)$ & $28,1 \pm 6,6$ & $26,8 \pm 4,6$ & 0,468 \\
\hline RER & $1,00 \pm 0,01$ & $1,00 \pm 0,01$ & 0,861 \\
\hline
\end{tabular}

Valores expressos em média+DP * ${ }^{*}<0,05$ vs SSM . SSM - grupo sem síndrome metabólica; CSM - grupo com síndrome metabólica; FCrep - frequência cardíca em repouso; FCmáx - frequência cardíaca máxima; FCrec - frequência

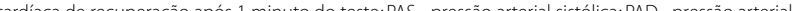
$\mathrm{VO}_{2 \text { pico }}$ - consumo de oxigênio pico no esforço máximo; RER - razão de troca respiratória.

Tabela 3. Índices de variabilidade da frequência cardíaca no domínio da frequência em repouso, durante teste cardiopulmonar de exercício e recuperação entre indivíduos classificados com e sem síndrome metabólica.

\begin{tabular}{c|c|c|c|c}
\hline \multirow{4}{*}{ Repouso } & & $\begin{array}{c}\text { SSM } \\
(\mathbf{n = 6 8 )}\end{array}$ & $\begin{array}{c}\text { CSM } \\
(\mathbf{n = 5 1 )}\end{array}$ & Valor de $\mathbf{p}$ \\
\hline & LF (nu) & $47,7 \pm 17,7$ & $50,2 \pm 17,7$ & 0,265 \\
\cline { 2 - 5 } & HF (nu) & $53,3 \pm 17,6$ & $49,8 \pm 17,6$ & 0,265 \\
\cline { 2 - 5 } & LF/HF & $5,5 \pm 5,2$ & $5,2 \pm 4,1$ & 0,265 \\
\hline \multirow{3}{*}{ TCPE } & LF (nu) & $59,8 \pm 12,2$ & $57,7 \pm 14,2$ & 0,407 \\
\cline { 2 - 5 } & HF (nu) & $40,1 \pm 12,2$ & $42,2 \pm 14,2$ & 0,407 \\
\cline { 2 - 5 } & LF/HF & $3,8 \pm 2,3$ & $3,5 \pm 2,3$ & 0,324 \\
\hline \multirow{3}{*}{ Recuperação } & LF (nu) & $63,3 \pm 14,6$ & $67,6 \pm 14,9$ & 0,639 \\
\cline { 2 - 5 } & HF (nu) & $33,7 \pm 14,6$ & $32,4 \pm 14,9$ & 0,639 \\
\cline { 2 - 5 } & LF/HF & $5,5 \pm 5,2$ & $5,2 \pm 4,1$ & 0,753 \\
\hline
\end{tabular}

Valores expressos em média $\pm D P . ~ * 0<0,05$ vs SSM. SSM -grupo sem síndrome metabólica; CSM - grupo com síndrome metabólica; LF -low frequency; HF-high frequency; LF/HF -razão entre low frequency e high frequency; TCPE - teste cardiopulmonar de exercício.

Tabela 4. Intervalos cardíacos em repouso, durante teste cardiopulmonar de exercício e recuperação entre indivíduos classificados com e sem síndrome metabólica.

\begin{tabular}{c|c|c|c|c}
\hline \multirow{4}{*}{ Repouso } & & $\begin{array}{c}\text { SSM } \\
(\mathbf{n = 6 8 )}\end{array}$ & $\begin{array}{c}\text { CSM } \\
(\mathbf{n = 5 1 )}\end{array}$ & Valor de $\mathbf{p}$ \\
\hline \multirow{4}{*}{ TCPE } & RRmin (ms) & $726 \pm 115$ & $703 \pm 99$ & 0,143 \\
\cline { 2 - 5 } & RRméd (ms) & $859 \pm 143$ & $819 \pm 119$ & 0,110 \\
\cline { 2 - 5 } & RRmáx (ms) & $999 \pm 173$ & $953 \pm 133$ & 0,174 \\
\hline & RRmin (ms) & $346 \pm 32$ & $350 \pm 44$ & 0,570 \\
\cline { 2 - 5 } & RRméd (ms) & $411 \pm 40$ & $416 \pm 47$ & 0,698 \\
\cline { 2 - 5 } & RRmáx (ms) & $522 \pm 73$ & $522 \pm 67$ & 0,907 \\
\hline \multirow{4}{*}{ Recuperação } & RRmin (ms) & $351 \pm 30$ & $359 \pm 40$ & 0,424 \\
\cline { 2 - 5 } & RRméd (ms) & $401 \pm 37$ & $408 \pm 51$ & 0,445 \\
\cline { 2 - 5 } & RRmáx (ms) & $458 \pm 50$ & $466 \pm 68$ & 0,710 \\
\hline
\end{tabular}

Valores expressos em média $\pm D P$. * $p<0,05$ vs SSM. SSM - grupo sem síndrome metabólica; CSM -grupo com síndrome metabólica; RR - intervalo entre ondas R; TCPE -teste cardiopulmonar de exercício.

\section{DISCUSSÃO}

No presente estudo objetivou-se analisar o comportamento da função autonômica cardiovascular em indivíduos com SM por meio da análise da VFC e foi possível observar que esse grupo de indivíduos apresenta valores para LF aumentados e HF reduzidos comparados a indivíduos sem a doença, embora não tenhamos encontrado evidências significativas. Vale ressaltar que mesmo que os indivíduos tenham sido classificados sem SM de acordo com a IDF, pelo menos um fator de risco para a SM estava presente, o que pode ter influenciado o fato de não termos encontrado diferença estatística entre os grupos.

Os índices de VFC no domínio da frequência em repouso, durante o TCPE e na recuperação entre os indivíduos não foram diferentes (Tabelas 3 e 4), fato que possivelmente constata que a presença de SM, definida de acordo com os critérios da IDF, não altera os índices de VFC. Similar aos nossos achados, Anaruma et al. ${ }^{14}$ estudaram 19 adultos divididos em dois grupos [grupo controle $(n=10)$ e grupo diabetes mellitus tipo $1(n=9)$ ] e também não encontraram diferenças nos valores de LF, HF e razão LF/HF nos momentos repouso, durante uma sessão de exercício aeróbico e na recuperação comparados a sujeitos sem a doença.

A FCrep foi similar entre os grupos, diferentemente de outros trabalhos que relataram maiores valores de FC basal no grupo com maior $I M C^{4,15}$. No entanto, merece atenção mencionar que no trabalho de Indumathy et al. ${ }^{4}$, que comparou 88 indivíduos divididos em dois grupos (obesos e não obesos), o $\Delta$ do IMC foi de $6,9 \mathrm{~kg} / \mathrm{m}^{2}$, o que difere do presente estudo que apresentou um $\Delta$ de $2,3 \mathrm{~kg} / \mathrm{m}^{2}$, o que possivelmente explica a ausência de diferença significativa. Lins et al. ${ }^{15}$ estratificaram 2.443 indivíduos em três grupos de acordo com os valores do IMC (normal: $18,5<\mathrm{IMC}<25$, sobrepeso: $25 \leq \mathrm{IMC}<30$, obeso: $\geq 30 \mathrm{~kg} / \mathrm{m}^{2}$ ) e observaram um $\triangle$ de 3,3bpm na FCrep entre os grupos normal e obeso, não encontrando diferenças significativas entre os grupos normal e sobrepeso. De forma interessante, o $\Delta$ da FCrep no presente trabalho foi de 3,2bpm, porém o $\triangle$ do IMC também foi menor comparado ao trabalho de Lins et al. ${ }^{15}$.

A PAS e PAD foram superiores no grupo CSM comparado ao SSM. E mesmo após a exclusão dos indivíduos que faziam uso de medicação para a PA no grupo SSM (dados não apresentados), as médias da PAS e PAD continuaram superiores no CSM (Tabela 2). De fato, há estudos que demonstram a associação positiva da hipertensão arterial à obesidade, especialmente a gordura central ou visceral, aumentando a resistência à insulina e a disfunção autonômica cardíaca ${ }^{16,17}$. Apesar do nível pressórico no grupo CSM ser mais elevado, não houve diferenças na VFC nos diferentes momentos investigados comparado ao grupo SSM. Trevizani et al. ${ }^{18}$ verificaram que o estresse autonômico imposto por uma sessão de exercício resistido não diferiu entre normotensos e sujeitos hipertensos com sobrepeso tratados com medicamentos e adicionalmente a VFC não foi diferente no repouso. Deve-se ressaltar que os indivíduos que faziam uso de betabloqueadores (drogas que influenciam diretamente no sistema nervoso autônomo) foram excluídos das análises.

A FCrec também é uma variável considerada como marcador da atividade vagal após um esforço físico 14,19,20. Não foram encontradas diferenças nos valores de FCrec após um minuto do fim do teste entre os

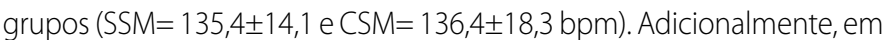
ambos os grupos durante o primeiro minuto da recuperação, os valores de LF aumentaram e de HF reduziram, o que corrobora dados similares previamente reportados ${ }^{14}$. $\mathrm{Grad}^{19}{ }^{19}$ conduziu teste em cicloergômetro em 46 pacientes divididos em dois grupos(com doença isquêmica cardíaca e sem a doença) objetivando comparar a FCrec após exercício e a relação com a VFC. Foi observado que a FCrec e a VFC reduziu não significativamente em pacientes isquêmicos e que pós-exercício a FCrec não foi correlacionada com os parâmetros de VFC medidos antes do exercício.

Os grupos não difeririam em relação à idade, mas o IMC foi superior no grupo CSM. Laederach-Hofmann et al. ${ }^{21}$ observaram o comportamento da função autonômica cardiovascular em indivíduos com sobrepeso/ obesidade divididos em três grupos segundo a faixa de IMC (27 a 32, 33 a 39, e acima de $40 \mathrm{~kg} / \mathrm{m}^{2}$ ). Após análise da VFC no domínio da frequência, observou-se que à medida que o IMC aumentava, o índice HF apresentava importante redução. Apesar de não havermos realizado estratificação dos indivíduos por faixas de IMC, uma vez que nossa amostra era composta por indivíduos classificados com SM e sem SM, nossos achados corroboram o encontrado por Laederach-Hofmann et al. ${ }^{21}$, uma vez que o grupo CSM apresentou maior IMC e índices de HF reduzidos comparados ao grupo SSM, embora não tenha ocorrido diferença estatística (Tabela 3). Como anteriormente citado, vale destacar que o $\triangle$ do IMC entre os grupos no trabalho de Laederach-Hofmann et al. ${ }^{21}$ foi de $5 \mathrm{~kg} / \mathrm{m}^{2}$ ou mais, diferente do encontrado em nosso estudo o que possivelmente explica a falta de 
diferença em relação aos índices deVFC. Ainda cabe ressaltar que Kurajoh et al. ${ }^{16}$ compararam 200 indivíduos divididos em dois grupos (diabetes e sem diabetes) e verificaram que a disfunção autonômica cardíaca em pacientes com diabetes está associada com obesidade visceral e hiperleptinemia. Os valores de glicemia de jejum foram superiores no CSM, mas estão abaixo do estado de pré-diabetes em ambos os grupos (Tabela 1), o que possivelmente explica a ausência de diferenças nos índices de VFC investigados.

São vários os fatores de risco para o desenvolvimento de doenças cardiovasculares, entre eles o aumento dos níveis de triglicérides, de colesterol total e de lipoproteína de baixa densidade (LDL-colesterol), diminuição de lipoproteína de alta densidade (HDL-colesterol), hipertensão, diabetes, tabagismo e inatividade física ${ }^{21,22}$. Entre os fatores de risco modificáveis, tais como fumo, obesidade e inatividade física ${ }^{23}, 0$ exercício físico promove adaptações autonômicas positivas, as quais podem ser evidenciadas pelo aumento da modulação parassimpática cardíaca ${ }^{24}$. Aeschbacher et al. ${ }^{25}$ sugerem que o estilo de vida pode ter um importante efeito positivo no sistema nervoso autônomo e que do total de 2079 indivíduos estudados, somente uma pequena minoria (3\% dos homens e $18 \%$ das mulheres) são classificados possuindo um estilo de vida saudável. Entre as variáveis observadas para a classificação, o nível de atividade física moderada (>150 min/semana) ou vigorosa (>75 min/semana) foi considerado. No presente trabalho, os indivíduos responderam ao Questionário Internacional de Atividade Física (IPAQ) e foram classificados como irregularmente ativos em ambos os grupos, fato que possivelmente explica a similaridade dos valores para o consumo pico de oxigênio $\left(\mathrm{VO}_{2 \text { pico }}\right)$ entre os grupos (Tabela 2$)$, uma vez que pessoas irregularmente ativas geralmente têm valores de $\mathrm{VO}_{2 \text { pico }}$ inferiores quando comparadas a indivíduos fisicamente ativos ${ }^{26}$.

\section{CONCLUSÕES}

Neste trabalho objetivamos comparar a VFC em indivíduos com e sem SM com a ideia de identificar parâmetros que possam auxiliar a realização de inferências a respeito da aptidão do sistema cardiovascular, de maneira rápida e simplificada. Com os resultados do presente estudo, pode-se concluir que a presença de SM, definida pelos critérios da IDF, parece não ser suficiente para provocar alterações nos índices de VFC em repouso, durante teste cardiopulmonar com exercício físico e na recuperação pós-teste quando os pacientes são comparados a indivíduos sem a doença. E comparando nossos dados com o da literatura ficou claro que apenas há piora nos parâmetros de VFC em níveis mais avançados da gravidade das doenças que constituem os critérios de SM.

\section{AGRADECIMENTOS}

Os autores agradecem a CAPES, o CNPq (processo 485045/2013-3) e a FAPESP (processo 2013-21159-8) pelo apoio dado a este trabalho e aos participantes que gentilmente colaboraram com este estudo.

Todos os autores declararam não haver qualquer potencial conflito de interesses referente a este artigo.

CONTRIBUICÕES DOS AUTORES: Cada autor contribuiu individual e significativamente para o desenvolvimento do manuscrito. JALR (0000-0002-4728-1925)*, GDF (00000001-8191-0652)* e IAF (0000-0001-6730-3039)* foram os principais contribuintes na redação do manuscrito e obtenção dos dados. JALR, IAF, AAT (0000-0001-6487-8160)* e LPF (0000-0003-1463-8155)* realizaram a análise e interpretação dos dados. JALR e CRBJ (0000-0002-2228-0758** avaliaram os dados da análise estatística. JALR, GDF e CRBJ realizaram a pesquisa bibliográfica, a revisão do manuscrito e contribuíram com o conceito intelectual do estudo. *ORCID (Open Researcher and Contributor ID).

\section{REFERÊNCIAS}

1. Wulsin LR, Horn PS, Perry JL, Massaro JM, D'Agostino RB. Autonomic Imbalance as a Predictor of Metabolic Risks, Cardiovascular Disease, Diabetes, and Mortality. J Clin Endocrinol Metab. 2015;100(6):2443-8.

2. Horwich TB, Fonarow GC. Glucose, obesity, metabolic syndrome, and diabetes relevance to incidence of heart failure. J Am Coll Cardiol. 2010;55(4):283-93.

3. Sant Anna Junior M, Carneiro JR, Carvalhal RF, Torres Dde F, Cruz GG, Quaresma JC, et al. Cardiovascular autonomic dysfunction in patients with morbid obesity. Arq Bras Cardiol. 2015;105(6):580-7.

4. Indumathy J, Pal GK, Pal P, Ananthanarayanan PH, Parija SC, Balachander J, et al. Association of sympathovagal imbalance with obesity indices, and abnormal metabolic biomarkers and cardiovascular parameters. Obes Res Clin Pract. 2015;9(1):55-66.

5. Stuckey MI, Kiviniemi AM, Petrella RJ. Diabetes and technology for increased activity study: the effects of exercise and technology on heart rate variability and metabolic syndrome risk factors. Front Endocrinol (Lausanne). 2013:4:121.

6. Kawaguchi LYA, Nascimento ACP, Lima MS, Frigo L, Paula Júnior AR de, Tierra-Criollo CJ, et al. Caracterização da variabilidade de frequência cardíaca e sensibilidade do barorreflexo em indivíduos sedentários e atletas do sexo masculino. Rev Bras Med Esporte. 2007;13(4):231-6.

7. Perrone-Filardi P, Savarese G, Scarano M, Cavazzina R, Trimarco B, Minneci S, et al. Prognostic impact of metabolic syndrome in patients with chronic heart failure: data from GISSI-HF trial. Int J Cardiol. 2015;178:85-90.

8. Tamburus NY, Paula RF, KunzVC, César MC, Moreno MA, da Silva E. Interval training based on ventilatory anaerobic threshold increases cardiac vagal modulation and decreases high-sensitivity c-reative protein: randomized clinical trial in coronary artery disease. Braz J Phys Ther. 2015;19(6):441-50.

9. Chintala KK, Krishna BH, N MR. Heart rate variability in overweight health 14 care students: correlation with visceral fat. J Clin Diagn Res. 2015;9(1):6-8.

10. Tjønna AE, Lee SJ, Rognmo $\varnothing$, Stølen TO, Bye A, Haram PM, et al. Aerobic interval training versus continuous moderate exercise as a treatment for the metabolic syndrome: a pilot study. Circulation. 2008;118(4):346-54

11. Vanderlei LCM, Pastre CM, Hoshi RA, Carvalho TD de, Godoy MF de. Noções básicas de variabilidade da frequência cardíaca e sua aplicabilidade clínica. Rev Bras Cir Cardiovasc. 2009;24(2):205-17.

12. Heart rate variability. Standards of measurement, physiological interpretation, and clinical use. Task Force of the European Society of Cardiology and the North American Society of Pacing and Electrophysiology. Eur Heart J. 1996;17(3):354-81.

13. Rognmo $\varnothing$, Hetland E, Helgerud J, Hoff J, Slørdahl SA. High intensity aerobic interval exercise is superior to moderate intensity exercise for increasing aerobic capacity in patients with coronary artery disease. Eur J Cardiovasc Prev Rehabil. 2004;11(3):216-22.

14. Anaruma CP, Ferreira M Jr, Sponton CH, Delbin MA, Zanesco A. Heart rate variability and plasma biomarkers in patients with type 1 diabetes mellitus: Effect of a bout of aerobic exercise. Diabetes Res Clin Pract. 2016;111:19-27.

15. Cristina T, Lins B, Valente LM, Celestino D, Filho S, Barbosa O. Relação entre a frequência cardíaca de recuperação após teste ergométrico e índice de massa corpórea. Rev Port Cardiol. 2015;34(1):27-33.

16. Kurajoh M, Koyama H, Kadoya M, Naka M, Miyoshi A, Kanzaki A, et al. Plasma leptin level is associated with cardiac autonomic dysfunction in patients with type 2 diabetes: HSCAA study. Cardiovasc Diabetol. 2015;14:117.

17. Lee JW, Lim NK, BaekTH, Park SH, Park HY. Anthropometric indices as predictors of hypertension among men and women aged 40-69 years in the Korean population: the Korean Genome and Epidemiology Study. BMC Public Health. 2015;15:140.

18. Trevizani GA, Peçanha T, Nasario-Junior O, Vianna JM, Silva LP, Nadal J. Cardiac autonomic responses afte resistance exercise in treated hypertensive subjects. Front Physiol. 2015;6:258.

19. Grad C. Heart rate variability and heart rate recovery as prognostic factors. Clujul Med. 2015;88(3):304-9

20. Tonello L, Reichert FF, Oliveira-Silva I, Del Rosso S, Leicht AS, Boullosa DA. Correlates of Heart Rate Measures with Incidental Physical Activity and Cardiorespiratory Fitness in Overweight Female Workers Front Physiol. 2016;6:405.

21. Laederach-Hofmann K, Mussgay L, Rúddel H. Autonomic cardiovascular regulation in obesity. J Endocrinol. 2000;164(1):59-66

22. Schroeder EB, Chambless LE, Liao D, Prineas RJ, Evans GW, Rosamond WD, et al. Diabetes, glucose insulin, and heart rate variability: the Atherosclerosis Risk in Communities (ARIC) study. Diabetes Care. 2005;28(3):668-74.

23. Thayer JF, Yamamoto SS, Brosschot JF. The relationship of autonomic imbalance, heart rate variability and cardiovascular disease risk factors. Int J Cardiol. 2010;141(2):122-31.

24. Nolan RP, Jong P, Barry-Bianchi SM, Tanaka TH, Floras JS. Effects of drug, biobehavioral and exercise therapies on heart rate variability in coronary artery disease: a systematic review. Eur J Cardiovasc Prev Rehabil. 2008;15(4):386-96.

25. Aeschbacher S, Bossard M, Ruperti Repilado FJ, Good N, Schoen T, Zimny M, et al. Healthy lifestyle and heart rate variability in young adults. Eur J Prev Cardiol. 2016;23(10):1037-44.

26. Grant CC, Murray C, Janse van Rensburg DC, Fletcher L. A comparison between heart rate and heart rate variability as indicators of cardiac health and fitness. Front Physiol. 2013;4:337. 